\title{
Gestaltung des demografischen Wandels durch Tarifvertrag?
}

Der 2006 vereinbarte „Demografie-Tarifvertrag“ in der Eisen- und Stahlindustrie startete unter äußerst schwierigen Bedingungen: Die Finanzkrise hat der Stahlindustrie einen massiven Einbruch von durchschnittlich 30 \% im Jahr 2009 erbracht, womit Probleme der Beschäftigungssicherung in den Vordergrund gerückt sind. Nichtsdestotrotz haben einige Unternehmen begonnen, die neuen Herausforderungen anzugehen, die sich mit Blick auf eine alternde Belegschaft stellen. Der Beitrag befasst sich mit der Umsetzung des Tarifvertrages und arbeitet in einem ersten Schritt die damit verbundenen betrieblichen Handlungsfelder heraus. Im zweiten Schritt wird nach den Möglichkeiten der Gestaltung des demografischen Wandels durch „offene Prozessnormen“ sowie nach den Chancen der Übertragbarkeit des „wachsenden Tarifvertrages“ auf andere Branchen gefragt.

OLAF KATENKAMP, HELMUT MARTENS, ARNO GEORG

\section{Einleitung}

Im Zuge des demografischen Wandels und angesichts zunehmend älter werdender Belegschaften ist es erforderlich, die Arbeitssituation in den Unternehmen alters- und alternsgerecht zu gestalten, um die Leistungs- oder Arbeitsfähigkeit über den gesamten Erwerbsverlauf hinweg zu erhalten. Die Arbeitsgestaltung wird damit - nicht nur aus gewerkschaftlicher Sicht - ein zentrales Feld des Alternsmanagements. Primäres Ziel der Arbeitsgestaltung ist es, Arbeit so zu gestalten, dass vorhandene Arbeitsbelastungen minimiert werden (oder gar nicht erst entstehen) und die Leistungs- und Innovationsfähigkeit der Mitarbeiterinnen und Mitarbeiter dauerhaft erhalten bleibt.

Die Forschung hat in den letzten beiden Dekaden das Thema „Demografie in der Arbeitswelt" forciert. Neben älteren Forschungsergebnissen, die im Zuge des Aktionsund Forschungsprogramms Humanisierung des Arbeitslebens ( $\mathrm{HdA}$ ) generiert worden sind, sind aktuell u. a. vier verschiedene Analyseperspektiven erkennbar:

- die Identifikation gesundheitsförderlicher und alternsgerechter Arbeitsgestaltung in speziellen Bereichen (z. B. in der Montage oder IT-Branche),

- Arbeitsanforderungen und Belastungen als Kumulation im Lebenslauf und in verschiedenen Altersgruppen,
- die altersgerechte Arbeitszeitgestaltung und die Auswirkungen der Verlängerung der Lebensarbeitszeit, - die Evaluation neuer „altersgerechter" Arbeitssysteme oder die Auswirkung der Arbeitsorganisation auf die Innovationsfähigkeit. ${ }^{2}$

Bäcker et al. (2009) konstatieren, dass die Unternehmen und deren Einfluss auf die altersspezifische Beschäftigung eine „black box“ seien (ebd., S. 280): Die Motive, die Ziele und die Anpassung der Maßnahmen im demografischen Wandel sind weitestgehend nicht bekannt. Zwar sind auch die globalen Folgen der Alterungsprozesse der Bevölkerung (oder im Betrieb die Alterszusammensetzung der Belegschaften) schwierig zu prognostizieren; gleichwohl überrascht nach Einschätzung der Autoren, dass angesichts der bereits eingetretenen und in der betrieblichen Realität sichtbaren Alterungsprozesse die Reaktionen der Unternehmen darauf nur lückenhaft nachvollzogen werden können. Es ist demnach „kaum bekannt, wie Betriebe auf

Vgl. z. B. das Projekt der Deutschen Forschungsgemein schaft (DFG): Altersdifferenzierte Arbeitssysteme (SPP 1184) mit 20 Teilprojekten.

(2) Vgl. hierzu den neuen Förderschwerpunkt des Bundesministeriums für Bildung und Forschung (BMBF): „Innovationsfähigkeit im demografischen Wandel“. 
den demographischen Wandel reagieren, welche Betriebe sich als reaktionsfähig erweisen und wodurch ein bewusster Umgang mit den Problemen, möglicherweise auch mit den Potenzialen, älterer Mitarbeiter hervorgerufen wird" (ebd. S. 280).

Angesichts der in der Öffentlichkeit, in Verbänden und Politik breit diskutierten Befürchtungen des „Mythos Demografie" und seiner Folgen wie Fachkräftemangel oder leere Rentenkassen, verwundert die Diagnose der „schwarzen Box“. Nachdenklich muss auch stimmen, dass trotz einer Vielzahl von geförderten Projekten eine große Lücke zwischen Theorie und Praxis zu beobachten ist. In einem Gutachten hat die PROGNOS AG (2010) den meisten Initiativen in Betrieben, Organisationen und öffentlichen Einrichtungen nur „befriedigende“ Noten (v. a. in Klein- und Mittelbetrieben - KMU's) ausgestellt: Oftmals fehlen Ressourcen und Kompetenzen oder die Projekte sind sehr kurzfristig angelegt. ${ }^{3}$ Integrative oder ganzheitliche Modelle, wie sie in der Forschung propagiert werden, sind in der Praxis die Ausnahme.

In den Befragungen zum demografischen Wandel stehen vor allem zwei Aspekte im Vordergrund: erstens, die Frage, ob das Thema bereits im Unternehmen angekommen sei („Sensibilisierung“), und zweitens, welche Maßnahmen und Instrumente eingesetzt werden. Beispielsweise zeigte das IfaA-Trendbarometer „Arbeitswelt“ im Herbst 2009 eine deutliche Zurückstufung des Themas „Demografischer Wandel“. Demografische Entwicklung, Belastungen im Arbeitsumfeld und Gesundheitsmanagement wurden von den befragten 400 Expertinnen und Experten im Vergleich zu anderen Themen wie Produktionsmanagement, Vergütungssysteme oder Arbeitssystemgestaltung in der Krisenzeit als weniger bedeutsam eingestuft.

$\mathrm{Zu}$ einem anderen Befund kommt das Projekt „Potenziale einer alternsgerechten Betriebs- und Tarifpolitik“, das - initiiert von der Initiative neue Qualität der Arbeit (INQA) - Untersuchungen in der Chemie- und Pharma-, der Metallund Elektroindustrie und im Einzelhandel umfasst. Hiernach wird der Bedarf von den Betrieben allmählich erkannt: $57 \%$ der befragten Betriebsräte $(n=289)$ haben es als $a k$ tuelles Thema für ihren Betrieb eingestuft (Tullius et al. 2011, S. 82) und $78 \%$ der Betriebsräte stufen es als wichtiges $\mathrm{Zu}$ kunftsthema ein. Die Personalmanager setzen den Handlungsbedarf etwas niedriger an (mit einem Drittel zu $55 \%$ in spe). Diese Schere in der Beurteilung der Dringlichkeit ist auch in der Stahlindustrie beobachtbar (vgl. Abschnitt 4). Die Zahlen belegen jedoch, dass das Thema insgesamt in den Betrieben angekommen ist.

Mit dem Tarifvertrag zum demografischen Wandel in der Eisen- und Stahlindustrie im Tarifbezirk NRWplus wurden erstmalig in einer Branche die Herausforderungen alters- und alternsgerechter Arbeit angegangen. Im Folgenden werden zuerst die gesellschaftlichen Rahmenbedingungen des demografischen Wandels kurz skizziert (Abschnitt 2), bevor die Hintergründe für den Abschluss des Tarifvertrages und seine zentralen Eckpunkte dargestellt werden (3). Abschnitt 4 zeigt Maßnahmen und zentrale Handlungsfelder in den Stahlunternehmen auf, die aus einer empirischen Studie mit einer repräsentativen (Wiederholungs-)Befragung und zehn Fallstudien resultieren. Abschließend wird die Eignung tarifpolitischer Instrumente zur Gestaltung des demografischen Wandels reflektiert (5).

\section{Demografischer Wandel als Herausforderung}

Der demografische Wandel wird in den fortgeschrittenen industriellen Gesellschaften die herkömmliche Alterspyramide zunehmend ins Ungleichgewicht bringen. Die Politik hat darauf zuerst mit einer Kehrtwende gegenüber der lange Zeit vorherrschenden Orientierung auf eine frühzeitige Ausgliederung Älterer reagiert: in Deutschland etwa mit den Rentenreformen von 1992 und 2001 (RiesterRente), den Hartz-Reformen (von 2003 bzw. 2004), mit der Gesundheitsreform sowie mit dem RV-Altersanpassungsgesetz $^{\oplus}$ (beide 2007). Man kann für die staatliche Politik der Konsolidierungsbestrebungen in der Finanzierung der Rentenversicherung, die nicht nur in Deutschland zu einer sukzessiven Heraufsetzung der Altersgrenzen für den Bezug von vorgezogenen Altersrenten geführt hat (Frerichs 2009; Burkert/Sproß 2010), von einer Strategie der Flankierung eines frühzeitigen Ausscheidens älterer Arbeitnehmerinnen und Arbeitnehmer aus Erwerbsarbeit hin zur stärkeren Integration älterer Menschen in das Erwerbsleben sprechen. Kritische Analysen von Politikansätzen, die neoliberalen Ursache-WirkungsZusammenhängen nicht folgen (Bosbach 2004), blieben unbeachtet. Auf der Ebene der Unternehmen wurden vor dem Hintergrund immer noch verbreiteter „Defizitmodelle“ des Alterns Konzepte zur Verjüngung von Belegschaften gefördert, womit der Blick auf sozial nachhaltige Konzepte einer alternsgerechten Arbeitsgestaltung eher verstellt wurde.

Die Gewerkschaften kritisieren die Heraufsetzung des Rentenalters auf 67 Jahre als faktisches Rentenkürzungs-

3 Vgl. das Prognos-Gutachten (2010): „Für die kleinen und mittleren Unternehmen, die häufig ohne Personalabteilung auskommen, ist hingegen nicht davon auszugehen, dass sie in der Breite über die Konsequenzen des demographischen Wandels informiert sind. Zwar befassen sich mehr und mehr KMU mit den genannten Herausforderungen, nach Einschätzung vieler Gesprächspartner jedoch wenig strategisch und zu kurzfristig." (S. 59)

(4) Der genaue Titel lautet: Gesetz zur Anpassung der Regelaltersgrenze an die demografische Entwicklung und zur Stärkung der Finanzierungsgrundlagen der gesetzlichen Rentenversicherung. 
programm. Der Tatbestand zunehmend längerer Lebenserwartungen und -arbeitszeiten ist aber gegeben, und in der Folge stellt sich für die Unternehmen die Herausforderung, Effizienz bei im Durchschnitt älteren Belegschaften zu sichern; aus Sicht der Beschäftigten geht es um die Frage nach den Chancen eines sinnhaft tätigen Lebens unter Vermeidung eines ,, arbeitsbedingten Voralterns“ vor allem vieler gewerblicher Beschäftigter (vgl. Ulich 2005). In den Unternehmen (IG Metall 2007; Bäcker et al. 2009) passiert im Hinblick auf demografische Herausforderungen vergleichsweise wenig. „Ein systematisches tarifpolitisches Konzept für eine altersgerechte Gestaltung der Arbeitsbedingungen (sei) erst noch zu entwickeln" forderte Bispinck vor zehn Jahren (2002, S.16). Inzwischen wurden nach der Stahlbranche auch in der Chemieindustrie und im Tarifbereich des Öffentlichen Personennahverkehrs (ÖPNV) Tarifverträge zur Gestaltung des demografischen Wandels abgeschlossen.

\section{Der Tarifvertrag in der Stahlindustrie}

Die Stahlindustrie ist eine der Branchen, die für die Entwicklung der industriellen Beziehungen in der Bundesrepublik Deutschland über lange Zeit eine prägende Rolle spielte - nicht nur wegen ihrer wirtschaftlichen Bedeutung, sondern aufgrund der Leitbildfunktion der Montanmitbestimmung. Im Zuge tief greifender technologischer Veränderungen der letzten 20 Jahre, begleitet von einer in quantitativer wie qualitativer Hinsicht massiven Personalanpassungspolitik, wurde der „lange Abschied vom Malocher" (Hindrichs et al. 2000) vollzogen. Das alte Leitbild angelernter Tätigkeiten und körperlicher Schwerarbeit ist heute nicht mehr prägend. Mittlerweile wird ein großer Teil der Arbeiten in der Produktion von Hüttenfacharbeitern und Verfahrensmechanikern in lärmgeschützten und klimatisierten Leitständen verrichtet. "Automationsarbeit ist der Kerntyp der neuen Arbeit in den modernen Stahlwerken“ (ebd., S. 30).

Die Stahlindustrie setzt verstärkt auf prozessorientierte Verfahren. Als Zulieferer für die Automobilindustrie oder Schifffahrt wird mehr High-Tech-Stahl (z.B. Oberflächenbehandlung) hergestellt. Die Anzahl der Facharbeiter ist deutlich gestiegen (fast $80 \%$ ) und der Anteil der ungelernten Kräfte in der Belegschaft hat sich seit 1990 halbiert. Traditionell ist in der Stahlindustrie die Schichtund Nachtarbeit sehr hoch (um die 75 \%). Die Umgebungsbelastungen sind mit $40 \%$ deutlich höher als in anderen Branchen, wobei der Anteil schwerer körperlicher Arbeit tendenziell zurückgeht. Die personalpolitische Bewältigung der Anpassungsprozesse erfolgte seit den 1980er Jahren vornehmlich im Wege einer konsequent betriebenen mitbestimmten Sozialplanpolitik der Arbeitsdirektoren („Alt geht, Jung bleibt"). Die Mitbestimmung bildet für die Pro- gramme zum demografischen Wandel weiterhin eine wichtige Plattform.

In der Stahlindustrie muss aber heute angesichts eines gegenüber der Gesamtindustrie um ca. zwei Jahre höheren Altersdurchschnitts eine Art Paradigmenwechsel - weg von den jungen und „olympiareifen“ Belegschaften, hin zur Integration älterer Beschäftigter - bewältigt werden.

Angesichts der gesellschaftlichen wie branchenspezifisch betrieblichen Konstellationen haben sich die Tarifpartner der Eisen- und Stahlindustrie 2006 auf einen Tarifvertrag zur Gestaltung des demografischen Wandels geeinigt.

Der Tarifvertrag zielt darauf ab

- die Arbeitsbedingungen alternsgerecht zu gestalten,

- die Gesundheit der Beschäftigten zu fördern, um ihre Beschäftigungsfähigkeit zu erhöhen,

- Wege zum vorzeitigen gleitenden Ausscheiden aus dem Arbeitsleben, insbesondere durch die (nicht zwingend vorgeschriebene) Einrichtung eines Fonds zur Gestaltung des demografischen Wandels, auch nach Fortfall der gesetzlichen Regelungen zur Altersteilzeit zu ermöglichen und - die Belegschaften in der Branche zu verjüngen.

Um eine Grundlage für weitere Aktivitäten auf betrieblicher Ebene zu schaffen, sollte in allen Betrieben und bezogen auf einzelne Organisationseinheiten spätestens neun Monate nach Inkrafttreten des Tarifvertrags (Juli 2008) in Abstimmung mit dem Betriebsrat mit der Erstellung einer Altersstrukturanalyse begonnen werden. Der Tarifvertrag sieht vor, dass Arbeitgeber und Betriebsrat sich gemeinsam auf Schlussfolgerungen verständigen, die aus der Altersstrukturanalyse zu ziehen sind, sowie die daraus resultierenden Maßnahmen einvernehmlich abstimmen. Der Betriebsrat hat zudem ein Vorschlagsrecht. Unterschiedliche Maßnahmen wie z. B. Gesundheitsförderung, Belastungswechsel und altersgemischte Teams oder Verkürzung der Lebensarbeitszeit werden beispielhaft aufgelistet.

In seinen Zielsetzungen ist der Tarifvertrag bemerkenswert offen. Einerseits sind die Erfahrungen des Qualifizierungstarifvertrages (vgl. Bahnmüller et al. 2004) eingegangen, andererseits schlagen sich hierin divergierende Interessen der Tarifparteien nieder (vgl. Georg et al. 2007, S. 38f.) wie auch der Umstand, dass über maßgerechte Instrumente der Problemlösung noch viele Unklarheiten bestanden - aufseiten des Managements wie auch der Gewerkschaft und der Betriebsräte. Vor diesem Hintergrund soll der Tarifvertrag „Leitplanken setzen“ und Prozesse in Gang bringen - er ist als ein „wachsender" Tarifvertrag angelegt (Brandl 2006, S. 131).

Nach einer Vorstudie (Georg et al. 2007) haben die Sozialforschungsstelle Dortmund und das Institut für Gerontologie der TU Dortmund einen zweifachen Zugang zur Empirie gewählt (vgl. Katenkamp et al. 2012): Zum einen wurden mittels einer standardisierten Panelbefragung von Betriebsräten und Personalmanagern im Wege von Telefoninterviews repräsentative Daten zur Umsetzung des 
Tarifvertrags erhoben. ${ }^{\ominus}$ Zum anderen wurden in zehn Betrieben nach „klassischem “ Muster ${ }^{\circledR}$ Fallstudien durchgeführt, um die jeweiligen Umsetzungsprozesse rekonstruieren und deren Ergebnisse hinreichend differenziert im Kontext je spezifischer Akteurs- und Bedingungskonstellationen beurteilen zu können. Die unterschiedlichen Erhebungsinstrumente führten zu in hohem Maße kongruenten Befunden. ${ }^{0}$

\section{Handlungsfelder und Maßnahmen in den Unternehmen}

Aus zahlreichen Studien kristallisieren sich diverse Handlungsfelder in der Praxis der Unternehmen heraus (vgl. Bäcker et al. 2009; Sporket 2011). In der Evaluationsstudie der Sozialforschungsstelle Dortmund und des Instituts für Gerontologie der TU Dortmund wurden sechs Handlungsfelder von den Akteuren betont: (1) Personalbeschaffung, (2) Qualifizierung und Personalentwicklung, (3) Gesundheitsförderung, (4) Alternsgerechte Arbeitszeitgestaltung und gleitender Ausstieg, (5) Arbeitsgestaltung und (6) Wissenstransfer. Bei den im Folgenden behandelten Aspekten geht es um einen summarischen Überblick der Umsetzungsergebnisse (für Details vgl. Katenkamp et al. 2012, S. 120ff. sowie S. 135ff.). Auf den unter den Bedingungen der Montanmitbestimmung in hohem Maße mitbestimmten und in der Regel unter aktiver Beteiligung der Betriebsräte sowie durch Unterstützungsmaßnahmen der IG Metall flankierten Umsetzungsprozess kann hier nicht vertiefend eingegangen werden (vgl. ebd. S. 86ff. sowie S. 126ff.). Etwa jedes zweite Unternehmen hat 2009 einen Lenkungskreis eingerichtet, alle Unternehmen haben eine Altersstrukturanalyse durchgeführt und im Schnitt wurden daraus je Betrieb vier bis sieben Maßnahmen abgeleitet.

\subsection{Personalrekrutierung}

Die Personalbeschaffung wird aus der Sicht der Personalabteilungen durchgängig als strategisch wichtiges Feld betrachtet. Während hinsichtlich der Qualifizierung von Facharbeitern in mittelfristiger Perspektive mit Problemen gerechnet wird, ist die Beschaffung von hoch qualifiziertem Personal (Ingenieure) für die Personalmanager schon heute eine Herausforderung. Die Zahl der auf Branchenbedarfe hin spezifisch qualifizierten Hochschulabgänger ist nicht sehr groß. Folgerichtig werden diverse Maßnahmen (Stipendien, Hochschulkontakte) ergriffen, um Personal zu rekrutieren.

\subsection{Qualifizierung}

Aber auch Maßnahmen der Personalentwicklung und Qualifizierung für Beschäftigung stehen im Kontext der Bewältigung des demografischen Wandels aus Sicht der Personal- manager stark im Vordergrund. Einerseits geht es darum, generell jüngere Beschäftigte, vor allem die Auszubildenden nach Abschluss ihrer Ausbildung, zu übernehmen. Andererseits nutzen Unternehmen das Instrument der Altersstrukturanalyse, um spezifische Ausbildungsbedarfe zu überprüfen. Drittens schließlich geht es um Fragen der Weiterbildung, etwa im Zuge der Einrichtung von Mehrbereichsarbeitsplätzen in der Produktion (vgl. Lichte 2008).

\subsection{Gesundheit}

Etwas vereinfachend kann man bei betrieblicher Gesundheitsförderung von einem ganzen Bündel von Maßnahmen sprechen, die allesamt darauf zielen, angesichts relativ alter Belegschaften im Bereich der Verhaltensprävention den negativen Folgen eines arbeitsbedingten Voralterns gegenzusteuern. Durchgängig finden sich hier Maßnahmen wie die Errichtung von Fitnesscentern, oder, wo sich dies wegen der örtlichen Gegebenheiten nicht anbietet, die Gewährung von Zuschüssen bei der Nutzung vorhandener Einrichtungen. Ferner wurden in mehreren Fällen konkrete Maßnahmen wie die Einrichtung von „Gesundheitsschichten“ oder die Durchführung von Rückenschulen usw. initiiert.

Insgesamt ist im Handlungsfeld Gesundheitsförderung in allen untersuchten Unternehmen eine relativ breite Palette von Maßnahmen ergriffen worden. Von den Betriebsräten wird allerdings kritisch angemerkt, dass z. B. eine Gesundheitsschicht im Abstand von drei Jahren schwerlich der Schlüssel zur Bewältigung der Herausforderungen einer fortschreitenden „Überalterung“ der Belegschaften sein könne und dass die Möglichkeit des Besuchs von Fitnesscentern von den ohnehin Aktiven, aber eben nicht von denjenigen Beschäftigten genutzt werde, die sportliche Aktivitäten am nötigsten hätten. Personal- und Gesundheitsmanager weisen hingegen darauf hin, dass solche Maßnahmen vor zehn Jahren in einigen Unternehmen undenkbar gewesen seien.

\subsection{Arbeitszeit}

In den Fallstudienbetrieben werden Fragen der alternsgerechten Arbeitszeitgestaltung als das gravierendste Problem bezeichnet, das einer Beschäftigung bis zum gesetzlichen Renteneintrittsalter entgegensteht. Veränderungen gelten

5 In der ersten Erhebungsrunde (Frühjahr 2009) mit 29 Personalmanagern und 35 Betriebsräten aus den 47 Betrieben im Tarifbezirk NRW plus, in der zweiten Runde (Spätherbst 2010) mit 16 Personalmanagern und 21 Betriebsräten.

6 Vgl. dazu allgemein: Pongratz/Trinczek 2010; im Detail zu unserer Untersuchung: Katenkamp et al. 2012, S. 39ff.

(7) Im Folgenden werden im Wesentlichen die Trendaussagen aus unseren Fallstudien präsentiert. Zu den Ergebnissen derTelefonbefragungen siehe im Einzelnen Katenkamp et al. 2012, S. 49ff. 
- im Sinne der nach dem Stand arbeitswissenschaftlicher Erkenntnisse derzeit besten, kurztaktigen und vorwärtsrollierenden Schichtmodelle ${ }^{\circledR}$ - als ausgesprochen schwierig, insbesondere, weil sie in die für Schichtarbeiter ohnehin aufwendige Gestaltung ihrer außerbetrieblichen Lebenszusammenhänge eingreifen und gewohnte Rhythmen verändern.

In etlichen Fällen ist daneben in Bezug auf bereits gesundheitlich beeinträchtigte Beschäftigte die Frage nach der Möglichkeit eines Ausscheidens aus dem Schichtbetrieb wichtig. In den Produktionsbereichen scheidet diese Möglichkeit aus arbeitsorganisatorischen Gründen aber aus. Nur in einem von zehn Fällen erfolgte in einem Instandhaltungsbereich die Einrichtung einer Tagschicht „aus demografischen Gründen“.

In vielen Fällen wird berichtet, dass man angesichts der nur schwer zu verändernden Verhältnisse im Hinblick auf bereits gesundheitlich beeinträchtigte Beschäftigte bemüht sei, im Wege von „Insourcing“-Maßnahmen die Zahl von sogenannten Schonarbeitsplätzen zu erhöhen. Allerdings sind die Spielräume hierfür begrenzt. Beim Wechsel auf Schonarbeitsplätze wird seitens der Betriebsräte sorgfältig darauf geachtet, entsprechende Schritte erst dann einzuleiten, wenn die an Alter und Dauer der Betriebszugehörigkeit festgemachten tariflichen Absicherungsbestimmungen voll greifen.

\subsection{Arbeitsgestaltung}

Seit „HdA-Zeiten“ gelten einzelne Unternehmen der Branche auf dem Gebiet der Arbeitsgestaltung als Vorreiter. In einzelnen Fällen wird berichtet, dass neue Arbeitsplatzanalysen unter dem Gesichtspunkt der altersgerechten Gestaltung von Arbeitsplätzen notwendig seien. Ingenieure, Ergonomen, Personalmanager und Betriebsräte betonen durchgängig, dass neue Arbeitsplatzanalysen ein ganz wesentliches Instrument seien, um zu einer demografiefesten Personalpolitik zu gelangen. Zum Zeitpunkt unserer Erhebungen (2010) standen entsprechende Schritte aber erst am Anfang, obwohl dieses Handlungsfeld neben dem der Arbeitszeitgestaltung als besonders wichtig angesehen wird. Insbesondere Betriebsräte berichten von zunehmenden psychischen Belastungen und Erkrankungen.

\subsection{Wissenstransfer}

Der Wissenstransfer wird angesichts des drohenden Verlustes von Erfahrungswissen infolge absehbaren massiven Ausscheidens der Alterskohorten der heute bereits über Fünfzigjährigen auch international als ein herausragendes Problem diskutiert (vgl. z. B. Warrian 2004; Katenkamp 2011). In der Stahlindustrie ist der Wissenstransfer aus Sicht der Personalmanager wie der Betriebsräte ein wichtiges Handlungsfeld, das frühzeitig im Umsetzungsprozess angegangen worden ist, aus Sicht beider Gruppen mit durchaus unterschiedlichem Erfolg. Beispielsweise werden Mehrbereichsarbeitsplätze, möglichst in altersgemischten
ÜBERSICHT 1

\section{Ausgewählte Aspekte in den Fallstudienbetrieben}

\begin{tabular}{l|c|c|c|c|c|c|c|c|c|c|c|c|}
\hline AspektelFallbetrieb & $\mathbf{1}$ & $\mathbf{2}$ & $\mathbf{3}$ & $\mathbf{4}$ & $\mathbf{5}$ & $\mathbf{6}$ & $\mathbf{7}$ & $\mathbf{8}$ & $\mathbf{9}$ & $\mathbf{1 0}$ \\
\hline Altersstruktur & A-A & A-A & A-A & K-A & A-A & K-A & K-A & B-B & K-B & A-A \\
\hline Rekrutierung & $* *$ & $* *$ & $* *$ & $* * *$ & $* *$ & $*$ & $* *$ & $* *$ & $* * *$ & \\
Qualifizierung & $*$ & $* *$ & $* *$ & $* * *$ & $*$ & $*$ & $* *$ & $*$ & $* * *$ & $* *$ \\
Gesundheit & $* * *$ & $* *$ & $* * *$ & $* * *$ & $* * *$ & $* *$ & $* *$ & $*$ & $* * *$ & $* *$ \\
\hline Arbeitszeit & $*$ & $* *$ & $* *$ & $*$ & $* *$ & $*$ & $* * *$ & $* *$ & $* * *$ & \\
\hline Arbeitsorganisation & $* *$ & & $* *$ & $* *$ & & & $*$ & $*$ & $* *$ & $*$ \\
\hline Wissenstransfer & $*$ & $* *$ & $* *$ & $* *$ & $* *$ & & $*$ & $*$ & $*$ & \\
\hline
\end{tabular}

1 Altersstruktur: Ist-Stand und Prognose 2020.

$\mathrm{A}=$ Alterszentriert, $\mathrm{K}=$ Komprimiert, $\mathrm{B}=\mathrm{Balancierte} \mathrm{Altersgruppen}$

* im Plan - ** eingeführt - *** aktiv (fortgeschritten), (bewertet wurden Anzahl, Umfang und Art der

Maßnahmen in diesem Feld), (vgl. die Details bei Katenkamp et al. 2012, Kap. 4.3. und 5.).

Quelle: Darstellung der Autoren auf Grundlage Katenkamp et al. 2012.

Gruppen, auf der Ebene qualifizierter Facharbeit oder langjährig angelernter Tätigkeiten als ein geeignetes Instrument aufgeführt. Mehrbereichsarbeitsplätze sind auch vermehrt eingerichtet worden, bei Weitem aber noch nicht flächendeckend. Tandembildungen von älteren Beschäftigten mit einem als Nachfolger vorgesehenen Beschäftigten gelten ebenfalls als sinnvoll. Einige Betriebsräte merken an, dass entsprechende Maßnahmen, letztlich aus Kostengründen, viel zu kurzzeitig erfolgten.

Zusammenfassend ist festzuhalten, dass Maßnahmen zur Personalbeschaffung und Personalentwicklung, zum Wissenstransfer sowie zur Gesundheitsprävention in aller Regel vorrangig verfolgt wurden und die „dicken Bretter“ der Arbeitszeit- und Arbeitsplatzgestaltung in einem zweiten Schritt angegangen werden sollen (Übersicht 1). Das mag damit zu erklären sein, dass ab Herbst 2008, d. h. mit Einsetzen der Finanzkrise, die jeweiligen Programme zur Gestaltung des demografischen Wandels ebenso wie die gewerkschaftlichen Aktivitäten zur Umsetzung des Tarifvertrags an Dynamik verloren haben. Gleichwohl überraschen einige Lücken in den Handlungsfeldern - gerade vor dem Hintergrund der berichteten HdA-Vorerfahrungen. Und selbst die mittlerweile gesetzlich vorgeschriebenen Gefährdungsanalysen sind nach Aussagen von Personalern und Betriebsräten in einigen Fällen noch nicht flächendeckend hinterlegt (vgl. Ahlers/Brussig 2004).

Aus Sicht beider Gruppen ist nach der bisherigen Umsetzung des Tarifvertrags noch ungeklärt, wie in der Branche zukünftig bis zum Ende des 67. Lebensjahres ge-

8 Vgl. die Beiträge zur IG-Metall Tagung „Ergonomische Schichtarbeit ist gut, weniger Schichtarbeit ist besser" zum Schwerpunktthema Arbeitszeit in: Gute Arbeit. Zeitschrift für Gesundheitsschutz und Arbeitsgestaltung 11/2011, S. 14 ff. 
arbeitet werden kann. Andererseits betonen $90 \%$ der Personaler und der Betriebsräte den Nutzen des Tarifvertrags. Trotz einiger unterschiedlicher Akzentsetzungen ist festzuhalten, dass es von beiden Seiten in hohem Maße übereinstimmende Bewertungen gibt. In der Telefonbefragung und in den Fallstudien wird die sensibilisierende, motivierende und aktivierende Wirkung des Tarifvertrages von beiden Seiten betont:

- Der Tarifvertrag habe zum richtigen Zeitpunkt wichtige Impulse gegeben,

- er gebe Orientierungen und sei zugleich weitgehend offen gehalten,

- zugleich erhöhe er den Handlungsdruck für beide Seiten.

Unterschiedliche Akzentsetzungen sind allerdings auch in diesen Gesamtbewertungen nicht zu übersehen: Die Personalmanager weisen eher darauf hin, dass der Tarifvertrag, gemessen am eigenen Unternehmensprogramm, doch nicht so viel „Neues“ gebracht habe - ausgenommen die viel diskutierten „Demografiefonds“ ${ }^{\oplus}$ Insgesamt haben zwei Konzerne (mit rund einem Dutzend Standorten von insgesamt 47 Unternehmen im Tarifbezirk NRWplus) Demografiefonds eingeführt. Betriebsräte betonen eher den Umstand, dass über den Tarifvertrag der Handlungsdruck für das Management erhöht worden sei. Ferner neigen sie dazu, eine Kann-Bestimmung des Vertrages als Soll-Bestimmung zu interpretieren, um ihren Forderungen im Alltag mehr Gewicht zu verleihen. Mehr als die Hälfte (56 \%) der Betriebsräte sind der Auffassung, dass der Tarifvertrag den Betrieb „wachgerüttelt“ habe, aber nur etwas mehr als jeder dritte Personaler ist dieser Ansicht. Betriebsräte sind engagiert und aktiv bei der Diagnose (Altersstrukturanalyse) und Initiative (Gremienbildung, bestimmte Handlungsfelder wie Arbeitszeit, Arbeitsorganisation) beteiligt. Auch Ideen zum Wissenstransfer werden mehrheitlich von den Betriebsräten geäußert. Auffällig ist, dass mehr Betriebsräte als Personaler sich an Schulungen und Qualifizierungen beteiligt haben. Den „Zahnlosen“ Tarifvertrag würden viele Betriebsräte gerne mit mehr Verbindlichkeit versehen: Einen Tarifvertrag mit konkreten Vorgaben wünschen sich $53 \%$ aller Betriebsräte (Stand: 2009), wohingegen nur $18 \%$ aller Personaler konkretere Vorgaben befürworten.

\section{Gestaltung des Demografischen Wandels durch Tarifvertrag?}

Im Organisationsbereich der IG Metall gibt es eine weit zurückreichende Tradition, mit Tarifverträgen Impulse für die sozialpolitische Gestaltung der Gesellschaft zu setzen. Dies ist verknüpft mit der Zeit der „aktiven Tarifpolitik“, d. h. der Phase überaus erfolgreicher Institutionalisierungen von Mitbestimmung, Tarifautonomie, prägenden Instituti- onen im Bereich sozialer Sicherung seit Mitte der 1950er Jahre und reicht bis in die 1970er Jahre hinein. Die Lohnfortzahlung im Krankheitsfall (1956) oder der Lohnrahmentarifvertrag II von 1973 sind in diesem Zusammenhang beispielhaft als Erfolge zu nennen.

Es ist klar, dass die Bedingungen einer solchen Tarifpolitik und Mitbestimmungspraxis heute angesichts der seither eingetretenen "Metamorphosen der sozialen Frage“ (Castel 2000) nicht mehr gegeben sind: Unter dem Druck einer entfesselten und globalisierten Ökonomie sind die Institutionen der alten „,institutionell verfassten Arbeitsgesellschaft" (v. Ferber 1961) fortschreitenden Erosionsprozessen ausgesetzt. Die Mitbestimmung war bis zum Beginn der großen Koalition im Jahr 2005 massiv unter Beschuss (Höpner 2004), und eine fortschreitende Erosion des Flächentarifvertrags ist nach wie vor nicht gestoppt, wenn auch die neue „betriebsnahe Tarifpolitik“ unter diesen Bedingungen immer noch unterschiedliche Gestaltungsoptionen eröffnet (vgl. Haipeter 2010).

Der Demografie-Tarifvertrag versucht, an die gewerkschaftliche Tradition gesellschaftspolitischer Gestaltung über tarifvertragliche Impulse anzuknüpfen. Betrachtet man die Umsetzungserfahrungen, ist zunächst unübersehbar, dass die Vorstellungen der IG Metall von einem „wachsenden“ Tarifvertrag mit dem gravierenden Problem konfrontiert worden sind, dass dieses „Wachsen“ in Zeiten „ausgeprägter Dürre“stattfinden musste. Anders als zum Zeitpunkt des Abschlusses von den Tarifparteien erwartet, wurden die seinerzeit recht optimistischen Wachstumsprognosen nicht nur durch eine normale zyklische Krise beeinträchtigt, sondern durch die weltweite Finanzkrise vollständig über den Haufen geworfen. Das Jahr 2009 stand im Zeichen von massiven Auftrags- und Produktionseinbrüchen, von lang dauernder Kurzarbeit in erheblichem Umfang und von vielfältigen Anstrengungen zum „Bau von Beschäftigungsbrücken“ (vgl. Bogedan et al. 2011). Mit dem Anziehen der Konjunktur im Folgejahr waren in der Branche auf den Beschaffungsmärkten massive Preisanstiege und auf den Absatzmärkten erhebliche Preiseinbrüche zu verzeichnen, sodass der Rationalisierungsdruck sich eher verstärkte.

Nach wie vor gilt aber, dass es im Hinblick auf die gestaltende Bewältigung des demografischen Wandels einen unverändert hohen Handlungsdruck gibt. In unterschiedlichem Maße mag zwar im Ergebnis der Krise das raschere Ausscheiden älterer Beschäftigter begünstigt worden sein, die drohende Überalterung der Belegschaften bleibt aber eine große Herausforderung, zumal die gesetzliche Unter-

(9 Ein betrieblicher „Demografiefonds" speist sich aus Mitteln der Arbeitnehmer und Arbeitgeber (in drei Fallbetrieben eine Gewinnbeteiligung - im Chemie-Tarifvertrag $300 €$ pro Mitarbeiter/Mitarbeiterin p.a.). Verwendet werden die Fondsmittel z. B. für die betriebliche Altersvorsorge, für Einzahlungen in Langzeitkonten oder für Altersteilzeit, die über den betriebsnotwendigen Bedarf hinausgehen. 
stützung von Altersteilzeitregelungen ausgelaufen ist, Arbeitsplätze für gesundheitlich beeinträchtigte ältere Arbeitnehmer rar sind und die Belastungen in der Arbeit (Schichtarbeit, zunehmende psychosoziale Belastungen, vgl. hierzu Meyn et al. 2011) eher zu- als abnehmen.

Vor diesem Hintergrund ist es zunächst als ein Umsetzungserfolg zu werten, dass das Thema des demografischen Wandels in allen Unternehmen auf der Agenda geblieben ist, auch wenn der Stellenwert der Umsetzung von Unternehmensprogrammen und/oder Tarifvertrag zwischen Herbst 2008 und Winter 2010 deutlich relativiert worden ist. Die Imperative des Standortwettbewerbs und des konzerninternen Benchmarkings erweisen sich für die Hoffnungen auf einen „wachsenden“ Tarifvertrag, der nachhaltig alternsgerechte Arbeitsbedingungen gestalten helfen soll, als in hohem Maße restringierend. Dass das Thema dennoch auf der Agenda blieb, verweist auf einen unveränderten Problemdruck in den Betrieben.

Nicht ganz leicht zu beantworten ist die Frage, ob der Tarifvertrag zum demografischen Wandel in der Eisen- und Stahlindustrie auf andere Bereiche in der Metall- und Elektroindustrie übertragbar ist bzw. als Vorbild dienen kann. Einerseits ist der Umstand zu beachten, dass die Unternehmen der Eisen- und Stahlindustrie aufgrund eines besonders hohen Durchschnittsalters der Belegschaften wie auch vergleichsweise schwieriger Arbeitsbedingungen v. a. aufgrund des extrem hohen Anteils von Kontischichtarbeit einen besonders großen Problemdruck verspüren. Andererseits sind eine im Branchenvergleich starke gewerkschaftliche Primärmacht und die im Betriebsalltag immer noch prägende Kraft der Montanmitbestimmung in Rechnung zu stellen. Dies alles dürfte von einiger Bedeutung dafür gewesen sein, dass betriebliche Umsetzungsprozesse nach den „Zeiten der Dürre“verstetigt werden konnten. Auffällig ist, dass der offene Charakter des Tarifvertrags und seine Ausrichtung auf Prozessnormen zunächst, also zu Beginn der Umsetzungsphase, von allen Beteiligten positiv bewertet worden sind. Dieser Zuschnitt wurde für die Suche nach und die Erprobung von geeigneten Lösungsansätzen als angemessen und hilfreich betrachtet. Drei Jahre später jedoch wurden die offenen Regelungen von vielen Betriebsräten als eine Schwäche des Tarifvertrags angesehen, weil aus ihrer Sicht damit eine deutliche „Schlagseite“ zu verhaltenspräventiven Maßnahmen befördert worden sei.

In Zukunft wird es um die Initiierung, Unterstützung und Stärkung von betriebsspezifischen Definitionen von Themen und Prozessen einer demographiefesten Personalpolitik gehen. Die drei bekanntesten Tarifverträge aus der Stahl- (NRWplus), der Chemieindustrie (bundesweit) und dem kommunalen ÖPNV (im Entwurf) unterscheiden sich in der Verbindlichkeit der Handlungsfelder, der Analyse der Alters- bis hin zur Reichweite der Gefährdungsanalyse und der Festlegung der einzelnen Maßnahmen. Während der Stahl-Tarifvertrag offen ist und eher auf neue Prozessnormen setzt, hat der Chemie-Vertrag einen hybriden Charakter: Er geht einen Schritt weiter und fußt auf drei Säulen: konkret werden Maßnahmen zur (1) alters- und gesundheitsgerechten Gestaltung, (2) zur Qualifizierung und (3) zur eigenen Vorsorge für gleitende Übergänge zwischen Bildung-, Arbeits- und Ruhestandsphasen fokussiert. Ein Demografie-Fonds mit $300 €$ pro Beschäftigten p.a. wird empfohlen, aber nicht verbindlich vorgeschrieben. Einen ganz anderen Charakter hat der Demografie-Tarifvertrag der kommunalen Arbeitgeberverbände und ver.di im öffentlichen Nahverkehr (VKA) (Entwurf): In sechs Bezirken sollen sowohl eine Demografieanalyse, Verwendung des Index Gute Arbeit als auch konkrete Qualifizierungsvereinbarungen (mind. fünf Qualifizierungstage, eine konkrete Quote für Auszubildende [FiF] im Verhältnis zu den regulär verrenteten und den vorzeitig wegen Krankheit ausscheidenden Mitgliedern eines Betriebes) verbindlich vorgeschrieben werden. Die Unternehmen sollen einen Gesundheitswert (Score-Verfahren) bilden, der über weitere Maßnahmen entscheidet.

Tullius et al. (2011) haben die drei Branchen Metall- und Elektroindustrie, Chemie und Einzelhandel hinsichtlich der Herausforderungen des demografischen Wandels für die Betriebe und Beschäftigen wie auch mit Blick auf Maßnahmen alternsgerechter Betriebs- und Tarifpolitik und deren Umsetzung verglichen. Sie konstatieren, dass trotz eines zum Teil hohen Aktivitätslevels in einzelnen Betrieben die „altersdifferenzierte Betriebspolitik ein Schattendasein“ (ebd. S. 208) fristet. Hierfür werden mehrere Gründe genannt: u.a. Pfadabhängigkeit (v. a. „Vorruhestandskultur“ ebd. S. 16), betriebliche "Gelegenheitsstrukturen“ (ebd. S. 15), „Interessensyndrom“ (ebd. S. 50), Spannungsverhältnis von kurzfristigen ökonomischen Interessen und langfristiger Demografiepolitik, fehlende Unterstützung auf der Führungsebene oder nicht konstruktiv-kooperatives Verhältnis von Betriebsräten und Personalverantwortlichen (vgl. ebd., Tab. auf S. 223). Die „Zerreißprobe“ der „normativen Kehrtwende“ (ebd. S. 54) durch das neue implizite Leitbild der Tarifpolitik (v. a. neue Erwerbsbiografie oder Lebenslaufpolitik, vgl. Naegele 2010) werden in den drei Branchen dargestellt. Gegenwärtig ist es aber (noch) zu früh, um abschließend zu beurteilen, ob und welche Tarifpolitik die nachhaltigste demografiegerechte Personalpolitik evoziert (ähnlich Tullius et al. 2011, S. 211). ${ }^{\circledR}$

Der Tarifvertrag in der Stahlindustrie war der erste seiner Art in der Bundesrepublik. Insofern stellt er eine Innovation im Tarifwesen dar. Die Stärke des Vertrages wird in seiner Offenheit gesehen: Er schreibt den Rahmen vor, nicht aber Details der Umsetzung. Die Akteure im Betrieb und die betriebliche Sozialorganisation sind ein zentraler Faktor bei der Realisierung: Die Arbeitsdirektoren haben in der Stahlindustrie strategisch sehr vorausschauend die

(10 Forschungstechnisch wäre eine mehrjährige Konfigurationsanalyse (im Sinne von Miebach 2009) für demografische Prozesse mit einer längeren Zeitdauer (z .B. zehn Jahre) sinnvoll. 
Herausforderungen des demografischen Wandels thematisiert. Die Betriebsräte haben zusammen mit den Personalern (ferner auch Arbeitsmedizinern) eine zentrale Rolle bei der Initiierung eingenommen. Betriebsräte haben vor allem eine Co-Management-Position bezogen: „Hartnäckige Konflikte“ traten nur selten auf. Reibungen waren insgesamt selten, da „das Thema zu wichtig“ sei (so unisono Betriebsräte und Personaler). In Einzelfällen gab es natürlich divergierende Interessen und Ansichten über Prioritäten - aber in Zeiten der Krise stand die Sicherung von Beschäftigung und der Standorte eindeutig im Vordergrund. Demografiegerechte Personalpolitik sollte aber keine „Schönwetterthematik“ sein. Die IG Metall und die IG BCE haben hierfür die ersten Anstrengungen unternommen: In beiden Branchen wird deutlich, dass die Neuorganisation einer alternsgerechten Arbeitspolitik kein Selbstläufer ist. Beide Tarifverträge konnten Impulse setzen - mehr nicht, aber auch nicht weniger! Der erste Schritt ist getan, weitere müssen folgen.

\section{LITERATUR}

Ahlers, E./Brussig, M. (2004): Gesundheitsbelastungen und Prävention am Arbeitsplatz - WSI-Betriebsrätebefragung 2004, in: WSI-Mitteilungen 57 (11), S. 617-624, http://www.boeckler.de/cps/rde/xbcr/hbs/wsimit_2004_11_ahlers. pdf

Bahnmüller, R./Fischbach, S. (2004): Der Qualifizierungsvertrag für die Metallund Elektroindustrie in Baden-Württemberg, in: WSI-Mitteilungen 57 (4), S. 182-189, http://www.boeckler.de/wsimit_2004_04_bahnmueller.pdf Bäcker, G./Brussig, M./Jansen, A./Knuth, M./Nordhause-Janz, J. (2009): Ältere Arbeitnehmer - Erwerbstätigkeit und soziale Sicherheit im Alter, Wiesbaden Bispinck, R. (2002): WSI-Tarifarchiv: Tarifpolitik für ältere Arbeitnehmerlnnen. Elemente qualitativerTarifpolitik, Düsseldorf

Bogedan, C./Brehmer, W./Seifert, H. (2011): Wie krisenfest sind betriebliche Bündnisse zur Beschäftigungssicherung?, in: WSI-Mitteilungen 64 (2),

S. 51-59, http://www.boeckler.de/wsimit_2011_02_Bogedan.pdf

Bosbach, G. (2004): Demografische Entwicklung nicht dramatisieren, in: Gewerkschaftliche Monatshefte 55 (2), S. 96-103

Brandl, S. (2006): „Deutsches Modell“ oder globalisiertes Arrangement? Transformation industrieller Beziehungen und soziale Nachhaltigkeit, Berlin

Burkert, C./Sproß, C. (2010): Früher oder später: Altersbilder auf Arbeitsmärkten im europäischen Vergleich, in: Brauer, K./Clemens, W. (Hrsg.): Zu Alt? "Ageism" und Altersdiskriminierung auf Arbeitsmärkten, Wiesbaden,

S. $149-170$

Castel, R. (2000): Die Metamorphosen der sozialen Frage. Eine Chronik der Lohnarbeit, Konstanz

Ferber, C. v. (1961): Die Institution der Arbeit in der industriellen Gesellschaft Versuch einer theoretischen Grundlegung. Habil.-Schrift, Göttingen (nur teilweise veröffentlicht)

Frerichs, F. (2009): Demografischer Wandel und Arbeitsmarktpolitik für ältere Arbeitnehmer/innen. Das Vereinigte Königreich, Japan und Deutschland im Wohlfahrtsstaatsvergleich, Berlin

Georg, A/Martens, H./Rockhoff, M./Sporket, M. (2007): Die Eisen- und Stahlin dustrie im demografischen Wandel, Dortmund, Forschungsbericht

Haipeter, T. (2010): Betriebsräte als neue Tarifakteure. Zum Wandel der Mitbestimmung beiTarifabweichungen, Berlin

Hans-Böckler-Stiftung (2009): Demografie im Wandel. Impulse für eine alternsgerechte Erwerbsarbeit, Düsseldorf

Hindrichs, W./Jürgenhake, U./Kleinschmidt, C./Kruse, W./Lichte, R./Martens, H (2000): Der lange Abschied vom Malocher. Sozialer Umbruch in der Stahlindus trie und die Rolle der Betriebsräte von 1960 bis in die neunziger Jahre, Essen Höpner, M. (2004): Unternehmensmitbestimmung unter Beschuss. Die Mitbestimmungsdebatte im Lichte der sozialwissenschaftlichen Forschung, MaxPlanck-Institut für Gesellschaftsforschung, MPIfG Discussion Paper 04/8, Köln
IG Metall (2007): Alternsgerechte Arbeit gestalten - Generationssolidarität organisieren. Arbeitshilfe zur Umsetzung desTarifvertrages zur Gestaltung des demografischen Wandels bei Eisen und Stahl, Düsseldorf

Katenkamp, O. (2011): Implizites Wissen in Organisationen, Wiesbaden Katenkamp, O./Martens, H./Georg, A./Naegele, G./Sporket, M. (2012): Nicht zum alten Eisen! Die Praxis des Demographie-Tarifvertrages in der Eisen und Stahlindustrie, Berlin

Lichte, R. (2008): Arbeit in der europäischen Stahlindustrie unter Veränderungsdruck, in: Arbeit 17 (2), S. 106-118

Meyn, C./Klatt, R./Georg, A. (2011): Psychische Belastungen in der Arbeitswelt in: Schröder, L./Urban, H. J.(Hrsg.): Gute Arbeit: Folgen der Krise - Arbeitsintensivierung, Restrukturierung, Frankfurt a. M., S. 249-260

Miebach, B. (2009): Prozesstheorie, Wiesbaden

Naegele, G. (2010): Grundlagen der sozialen Lebenslaufpolitik, Wiesbaden

Pongratz, H. J./Trinczek, R. (Hrsg.) (2010): Industriesoziologische Fallstudien. Entwicklungspotentiale einer Forschungsstrategie, Berlin

PROGNOS AG (2010): Recherche und Darstellung betrieblicher Demographieprojekte, unveröffentl. Gutachten für die BAuA, Dortmund

Sporket, M. (2009): Alternsmanagement in der betrieblichen Praxis. Motive, Umsetzung, Effekte, in: Zeitschrift für Gerontologie 42 (4), S. 292-298

Sporket, M. (2011): Organisationen im demographischen Wandel - Alternsmanagement in der betrieblichen Praxis, Wiesbaden

Tullius, K./Grabbe, J./Freidank, J. (2011): Altersdifferenzierte und alternsgerechte Betriebs- und Tarifpolitik zur Förderung der Innovations- und Beschäftigungssicherung Älterer, INQA-Projekt 03-08, unveröffentl. Abschlussbericht, Kassel/Göttingen

Tullius, K./Freidank, J./Grabbe, J/Kädtler, J./Schroeder, W. (2012): Perspektiven alter(n)sgerechter Betriebs- und Tarifpolitik; in: WSI-Mitteilungen 65 (2), S. 113-123, http://www.boeckler.de/wsimit_2012_02_tullius.pdf

Ulich, E. (2005): Arbeitspsychologie, Stuttgart

Warrian, P. (2004): Measuring Up in Steel: Performance Measurement and Innovation Policy in the Canadian Steel Industry, in: International Review of Administrative Sciences (70) 1, S.137-155

\section{AUTOREN}

OLAF KATENKAMP, Dr., ist wissenschaftlicher Mitarbeiter der Sozialforschungsstelle (sfs) Dortmund, Technische Universität Dortmund. Arbeitsschwerpunkte: Wissensmanagement, Innovation, Demografischer Wandel Arbeits- und Gesundheitspolitik.

katenkamp@sfs-dortmund.de

HELMUT MARTENS, Dr., ist Politikwissenschaftler und war bis zum März 2012 an der sfs Dortmund tätig. Arbeitsschwerpunkte: Mitbestimmung, Gewerkschaften und Arbeitspolitik.

martens@sfs-dortmund.de

ARNO GEORG, ist Koordinator des Forschungsbereichs Arbeitspolitik und Gesundheit der sfs Dortmund. Arbeitsschwerpunkte: Arbeit und Gesundheit.

georg@sfs-dortmund.de 